\title{
To Assess the Influence of Habitual Mouth Breathing and its Associated Taste Alterations
}

\author{
Sanghera SK ${ }^{1}$, Singh $\mathrm{CS}^{2}$, Naib T' ${ }^{1}$, Singh $\mathrm{B}^{1}$ and Singh $\mathrm{H}^{3 *}$ \\ ${ }^{1}$ BDS, SGRD Dental College Amritsar, India \\ ${ }^{2}$ BDS, Desh Bhagat Dental College and Hospital, India \\ ${ }^{3}$ Department of Oral Medicine and Radiology, SGRD Dental College Amritsar, India
}

* Corresponding author: Haramandeep Singh, Associate Professor, Department of Oral Medicine and Radiology, SGRD Dental College Amritsar, Punjab, India, Email: Mediteon20@gmail.com

\section{Research Article \\ Volume 5 Issue 4}

Received Date: October 07, 2020

Published Date: October 23, 2020

DOI: $10.23880 /$ oajds-16000274

\section{Abstract}

Background: If the sense of taste is impaired, it may often negatively affect patient's food intake, nutritional status and consequently health condition because it provides information about the food we eat.

Aim and Objective: To study the influence of habitual mouth breathing and its associated taste alterations and also investigate the cause of this influence.

Method and Methodology: Total eighty subjects were taken out of which forty healthy volunteers (male-26; female14) participated in this study. The mean age of these subjects was 25.6 1.9 (SD) years (age range, 19-36 years). Furthermore forty subjects (male-21; female-19) with a habit of mouth breathing (MB) were taken. The mean age of these subjects was $29.5 \pm 3.4$ (SD) years (age range, 19-40 years). A taste threshold is the minimum concentration at which taste sensitivity to a particular substance or food can be perceived. The subjects were told to avoid eating or drinking anything, with the exception of water for 3 hours before evaluation. In addition, a complete Ear, Nose, Throat examination was performed. We carried out the chemical method of gustatory testing by using filter paper taste strips. This is a validated gustatory test, used to measure the gustatory sensitivity of the tongue for 5 tastants; sweet, sour, salty, bitter, and umami. We measured the recognition thresholds only on one side on the tongue either left or right. There are many factors that can alter taste senstivity. Considering these factors can help us better understand and ensure accurate sensory taste perception. Although there are a variety of factors but some that influence taste perception more than others are discussed in this study. These various effects are brought about by the many organic and inorganic constituents of saliva. In response to these variations in saliva, taste sensitivity may also fluctuate widely.

Conclusion: Relationships between individual salivary constituents and certain taste substances have been explored in human psychophysical and animal experiments. However, there are many organic and inorganic salivary constituents whose effects on taste acuity have not yet been fully elucidated

Keywords: Mouth breathing; Taste alterations

Abbreviations: MB: Mouth Breathing.

\section{Introduction}

Taste is one of the major of basic five senses. It is a matter of survival and not merely for pleasure of flavours. If the sense of taste is impaired, it may often negatively affect patient's food intake, nutritional status and consequently health condition because it provides information about the food we eat. This taste sensation helps us differentiate stale from fresh food but taste sensation is also accompanied by smell sensation in its job. Breathing however when 
obstructed due to various reasons can lead to alterations in taste sensation. When the nasal airway is obstructed because of rhinitis or obstruction due to other cause we are forced to breathe through mouth [1]. Mouth breathing is defined as habit of respiring through only the mouth or through mouth and nose for longer than 6 months [2]. There are various causes that lead to mouth breathing in a person like nasal congestion, enlarged adenoids, enlarged tonsils, deviated septum, nasal polyps, shape and size of jaw etc. It is associated with disorders such as abnormal orofacial development, dry mouth, malocclusion, tooth caries, periodontal diseases, and oral malodour [3]. Nasal obstruction is also a potential risk factor for obstructive sleep apnea syndrome [4]. Some studies suggest dependence of taste sensation on genetics although the results have not been completely established. Genes that affect energy metabolism rather than taste and smell might be more powerful determinants of food intake. For instance, the different feelings of satiety and hunger that people experience arise from genetic variation [5]. Taste is closely associated and the smell mechanism arouses the taste receptors, placing a big influence on the latter, probably explaining why the oral-breathing individuals show deviations of the nutritional status [6]. When breathing through the mouth, it cannot be detected the taste and aroma of the food; therefore, a choice for food is not made by appetite but by the food consistency and easiness to swallow it [7]. However, the influence of habitual MB on taste perception was scarcely examined, though one study investigated the influences of various factors including MB on taste thresholds and reported that MB was not an influential factor [8].
Therefore it was interesting to study the influence of habitual mouth breathing and its associated taste alterations and also investigate the cause of this influence.

\section{Materials and Methods}

\section{Subjects}

Total eighty subjects were taken out of which forty healthy volunteers (male-26; female14) participated in this study. The mean age of these subjects was $25.6 \pm 1.9$ (SD) years (age range, 19-36 years). Furthermore forty subjects (male-21; female-19) with a habit of mouth breathing (MB) were taken. The mean age of these subjects was 29.5 3.4 (SD) years (age range, 19-40 years). We excluded subjects with current or past conditions that might be related to gustatory dysfunction (head traumas, infections of the upper respiratory tract, exposure to toxic substances, middle ear surgeries and intake of daily medication which could affect gustatory function). Young subjects were preferably recruited for this study to ensure a homogenous group of participants [9]. Subjects were classified into two groups the mouth- breathing (MB) and control groups-on the basis of their answers to the three questions shown in Table 1 which had been used to identify mouth breathers during daytime [10]. Subjects chose 0-1 and 2-3 from among the underlined options were considered as nasal and mouth breathers, respectively. Additional questionnaire included questions regarding age, sex, smoking habits, drinking habits and taste preferences as well as problems such as dry mouth, nasal obstruction, snoring and olfaction Table 2.

\begin{tabular}{|c|c|c|c|c|}
\hline Questions & \multicolumn{4}{|c|}{ Choices } \\
\hline How do you breathe usually? & Nose usually & Mouth usually & Mouth only & Nose and mouth \\
\hline Is your mouth open? & Usually closed & Sometimes open & Often open & Always open \\
\hline Is your mouth open when chewing? & Usually closed & Usually open & Both & \\
\hline
\end{tabular}

Note: Subjects who chose $0-1$ and 2-3 out of the underlined options were regarded as nasal and mouth breathers, respectively.

Table 1: Questionnaire for Identification of mouth breathing habit.

\begin{tabular}{|c|c|c|c|c|}
\hline Questions & & $\begin{array}{c}\text { Control group } \\
\mathbf{n}(\%)\end{array}$ & $\begin{array}{c}\text { MB group } \\
\text { n(\%) }\end{array}$ & Fishers exact test P-value \\
\hline \multirow{2}{*}{ Sex } & Male & $8(53.3 \%)$ & $10(66.7 \%)$ & \multirow{2}{*}{0.710} \\
\hline & Female & $7(46.7 \%)$ & $5(33.3 \%)$ & \\
\hline \multirow{2}{*}{ Are you a current smoker? } & Yes & $0(0.0 \%)$ & $1(6.7 \%)$ & \multirow{2}{*}{1.000} \\
\hline & No & $15(100.0 \%)$ & $14(93.3 \%)$ & \\
\hline \multirow{2}{*}{ Do you drink alcohol habitually? } & Yes & $6(40.0 \%)$ & $8(53.3 \%)$ & \multirow{2}{*}{0.715} \\
\hline & No & $9(60.0 \%)$ & $7(46.7 \%)$ & \\
\hline \multirow{2}{*}{ Do you like strong tastes? } & Prefer & $5(33.3 \%)$ & $12(80.0 \%)$ & \multirow{2}{*}{$0.025^{*}$} \\
\hline & Not Prefer & $10(66.7 \%)$ & $3(20.0 \%)$ & \\
\hline
\end{tabular}




\begin{tabular}{|c|c|c|c|c|}
\hline \multirow{2}{*}{ Do you ordinarily open your mouth? } & Yes & $3(20.0 \%)$ & $15(100.0 \%)$ & \multirow{2}{*}{$<0.001^{*}$} \\
\hline & No & $12(80.0 \%)$ & $0(0.0 \%)$ & \\
\hline \multirow{2}{*}{ Do you ordinarily feel mouth dryness? } & Yes & $2(13.3 \%)$ & $10(66.7 \%)$ & \multirow{2}{*}{$0.008^{*}$} \\
\hline & No & $13(86.7 \%)$ & $5(33.3 \%)$ & \\
\hline \multirow{2}{*}{ Do you have nasal obstruction? } & Yes & $2(13.3 \%)$ & $11(73.3 \%)$ & \multirow{2}{*}{$0.003^{*}$} \\
\hline & No & $13(86.7 \%)$ & $4(26.7 \%)$ & \\
\hline \multirow{2}{*}{ Do you have difficulty in smelling? } & Yes & $4(26.7 \%)$ & $6(40.0 \%)$ & \multirow{2}{*}{0.700} \\
\hline & No & $11(73.3 \%)$ & $9(60.0 \%)$ & \\
\hline \multirow{2}{*}{ Do you snore during sleep? } & Yes & $3(20.0 \%)$ & $10(66.7 \%)$ & \multirow{2}{*}{0.025} \\
\hline & No & $12(80.0 \%)$ & $5(33.3 \%)$ & \\
\hline
\end{tabular}

MB: Mouth breathing; ${ }^{*} \mathrm{P}<.05$.

Table 2: Subject characteristics.

\section{Measurement of Recognition Threshold for Five Basic taste}

A taste threshold is the minimum concentration at which taste sensitivity to a particular substance or food can be perceived. The subjects were told to avoid eating or drinking anything, with the exception of water for 3 hours before evaluation. In addition, a complete Ear, Nose, Throat examination was performed. We carried out the chemical method of gustatory testing by using filter paper taste strips. This is a validated gustatory test, used to measure the gustatory sensitivity of the tongue for 5 tastants; sweet, sour, salty, bitter, and umami. We measured the recognition thresholds only on one side on the tongue either left or right. We took filter papers and soaked them in a test solution. The solution was made by an 8 stepwise, two-fold dilution series of five basic tastes; sucrose (sweet), citric acid (sour), $\mathrm{NaCl}$ (salty), Quinine hydrochloride(bitter), and monosodium L-glutamate(umami). We placed the filter papers on the tongue region for about 3-4 seconds. The placement of the filter paper was placed away from the midline to selectively stimulate one branch of the chorda tympani on the tip and the glossopharyngeal around the root of the tongue. As mentioned above, solutions were each prepared at eight different concentrations. For each solution tested, the participants chose one of the following six possible answers: sweet, salty, umami, sour, bitter or no taste. If the patient could not correctly identify the taste, in the next trial, we used the solution in the same taste category with the next higher concentration. If the patient answered correctly, we tested a different taste solution. Before testing another solution, the patients rinsed their mouths thoroughly with deionized water until the previous taste disappeared. Once the subject could correctly identify the taste quality it was defined as the recognition threshold. When a subject failed to recognize a taste at the highest concentration, the matching recognition threshold was expressed as [9]. We applied the taste solutions in the following order; sweet, salty, umami, sour, and bitter. We did this to ensure that the bitter taste, which tends to remain in the oral cavity for a long time, was applied last (Table 3).

\begin{tabular}{|c|c|c|c|c|c|}
\hline $\begin{array}{c}\text { Concentration } \\
\text { step }\end{array}$ & $\begin{array}{c}\text { Sweet Sucrose } \\
\text { (M) }\end{array}$ & $\begin{array}{c}\text { Sour Citric } \\
\text { acid (M) }\end{array}$ & $\begin{array}{c}\text { Salty NaCl } \\
\text { (M) }\end{array}$ & $\begin{array}{c}\text { Umami Monosodium } \\
\text { glutamate (M) }\end{array}$ & $\begin{array}{c}\text { Bitter Quinine } \\
\text { hydrochloride (mM) }\end{array}$ \\
\hline 8 & 0.5 & 0.03 & 0.3 & 0.4 & 0.2 \\
\hline 7 & 0.25 & 0.015 & 0.15 & 0.2 & 0.1 \\
\hline 6 & 0.125 & 0.0075 & 0.075 & 0.1 & 0.05 \\
\hline 5 & 0.0625 & 0.00375 & 0.0375 & 0.05 & 0.025 \\
\hline 4 & 0.03125 & 0.001875 & 0.01875 & 0.025 & 0.0125 \\
\hline 3 & 0.015625 & 0.000938 & 0.00938 & 0.0125 & 0.00625 \\
\hline 2 & 0.007813 & 0.000469 & 0.00469 & 0.00625 & 0.001563 \\
\hline 1 & 0.003906 & 0.000234 & 0.00234 & 0.003125 & 0.003125 \\
\hline
\end{tabular}

Table 3: Substances and concentration steps of five basic taste solutions used for measurement of recognition threshold. 


\section{Open Access Journal of Dental Sciences}

\section{Measurement of Factors Relevant to Taste Senstivity}

There are many factors that can alter taste senstivity. Considering these factors can help us better understand and ensure accurate sensory taste perception. Although there are a variety of factors but some that influence taste perception more than others are discussed in this study.

Temperature: Influence of temperature on taste is not uniform. Taste buds can be put out of action by both high and low temperatures. Increasing temperature appears to increase the response to sweetness and decrease it to saltiness and bitterness. Decreasing temperature appears to increase the response to bitterness and decrease the response to sourness [11].

Obesity: Children and adolescents who are obese have less sensitive taste buds. That means for obese children, sweet foods taste less intensely sweet, bitter foods are milder and salt is not as readily perceived [12].

Disease: People with cancer and anorexia have reduced taste sensitivity as the result of their compromised physical condition. Cancer patients have reported that taste changes return to normal after treatment is completed [13].

Pregnancy: During pregnancy, nearly two thirds of women experience changes in taste. Pregnant women have been found to have a reduced sensitivity to salty tastes, which may be the body's way of ensuring increased salt intake during pregnancy [14] and other factors like adaptation, food medium, smoking, hunger are also responsible in altering taste sensitivity.

To analyse factors relevant to taste sensitivity we examined unstimulated and stimulated salivary flow and spinnbarkeit (viscosity), oral moisture level, and olfactory function.

\section{Measurement of Salivary Flow Rate}

Unstimulated salivary flow: The subjects were asked to swallow to void the mouth of saliva. Patient is asked to relax and keep minimal body movements, head leaned a little forwards and mouth slightly open over the mouth of funnel. At the end of collection period (1minute) the patients are asked to spit any remaining saliva into the test tube after which the weight of the collected saliva $(\mathrm{g} / \mathrm{min})$ was measured.

Stimulated salivary flow: The patients were instructed to swallow to void the mouth of saliva. Patient is asked to chew on an inert gum base and after 1 minute asks them to spit in funnel and keep chewing. Repeat the process again but discard the first collection, guide patient to spit and both saliva and gum at the end of collection period. Eliminate the gum before weighing the collected saliva.

Salivary spinnbarkeit: The spinnbarkeit of unstimulated saliva was measured using Neva Meter (Ishikawa Ironworks Corp., Kitakyusyu, Fukuoka, Japan). This machine measures the length at which a thread of stretched saliva snaps. The longer the length, the higher the viscosity of saliva..

\section{Discussion}

The habit to breathe through the mouth instead of the nose is an exclusively topical problem nowadays. Despite the development of medicine and contemporary early diagnostic tools, the percentage of mouth breathing children and some adults grows. Although taste sensation has numerous factors that affect the perception but the specific study about effect of mouth breathing has not been investigated enough. In this article we tried studying recognition thresholds for five basic tastes in mouth breathing and control groups. For identifying subjects with MB habit in accordance with the criteria of Yamaguchi, et al. [15], we used the questionnaire shown in Table 1.

Keeping the subjects of almost same age, habit of smoking, alcohol consumption etc the main result obtained was that there was higher threshold for some tastes in mouth breathing group than in control group. The results showed how threshold for all the tastes except salty and umami tastes were higher in mouth breathing group than in control group and there was not significant difference in salty or umami taste. Different studies using molecular and genetic approaches have identified several GPCRs as taste receptors. The attractive tastes, sweet and umami, are sensed by heterodimeric GPCRs assembled by the combinatorial arrangement of taste receptor type 1 subunit 1 (T1R1), taste receptor type 1 subunit 2 (T1R2), and taste receptor type 1 subunit 3 (T1R3) subunits. T1R1 and T1R3 form a complex to act as the umami taste receptor, whereas T1R2 and T1R3 form a complex to act as the sweet taste receptor. GPCRs of the T2R family were identified as bitter taste receptors, while the proton conductance and the $\mathrm{K}+$ channel enable amplification of sensory to sour stimuli. The epithelial sodium channel was proposed to act as a salty taste receptor candidate. Only a single type of taste receptor is expressed by a single taste cell; taste information is processed for each taste quality at the level of peripheral taste cells. Downstream signaling of taste receptors in type II cells involves $\alpha$-gustducin and phospholipase C-beta 2 (PLC $\beta 2$ ), which cause cell depolarization. When tastants bind to the T1R2-T1R3 receptor, conformational changes occur in the receptor, causing the activation of a series of signal transducers such as $\alpha$-gustducin or PLC $\beta 2$, pointing to the crucial role of these molecules in transducing tastant information. In humans, suppression of T1R3 gene expression induced hypoperception of umami and sweet tastes, suggesting that decreased expression of taste-associated genes such as T2R may be involved in hypogeusia. Since nasal obstruction is the 


\section{Open Access Journal of Dental Sciences}

main cause of mouth breathing, human taste sensing may be adversely affected by nasal obstruction [16].

Comparison of taste thresholds at the tip of the tongue (fungiform papillary region) revealed significantly higher thresholds for sweet and sour tastes in the MB group than in the control group. The reason why such differences were not observed in the thresholds for salty, umami, and bitter tastes might be that these tastes exhibited wider distributions of thresholds compared with sweet and sour tastes. At the root of the tongue (circumvallate papillary region), the thresholds for sour and bitter tastes were significantly higher in the MB group than in the control group. In order to analyse the reason for intergroup differences in taste sensitivity, we further investigated factors relevant to taste function, such as salivation, oral moisture, and olfactory function. In addition, we also conducted a self-administered questionnaire study. Saliva plays an essential role in maintenance of taste sensitivity. It was also seen that the people in mouth breathing group have a preference for strong tastes. The reason could be them feeling the food more tasteless most of the time with less flavour is why they preferred strong tasted. This could be a similar phenomenon that diabetics have a blunted sweettaste response [10] and a desire for high carbohydrate foods. The dehydration caused by oral breathing is likely to change the structure of the papillae or taste buds on the tongue. Some studies have indicated that the taste buds in the circumvallate papillae appeared shrunken and disorganized in rats that underwent desalivation [17]. Hence when olfactory senses get affected there is an decreased perception of taste. Infact many patients complaining of taste deterioration exhibited a decline in olfactory function rather than in taste function. It is seen that in majority of patients mouth breathing is a consequence of some nasal obstruction or facial deformity, nasal obstruction has been shown to induce changes in the physiology, form, and function in the craniofacial area. The findings in the present study suggest that nasal obstruction might have significant influences on gustatory function via morphologic changes in the taste papillae and taste buds of the tongue [18-21].

\section{Conclusion}

Saliva is seen to be really important factor in taste perception. Saliva affects taste sensitivity in various ways such as through diffusion of taste substances, chemical interaction with taste substances, stimulation of taste receptors, and protection of taste receptors. These various effects are brought about by the many organic and inorganic constituents of saliva. In response to these variations in saliva, taste sensitivity may also fluctuate widely. Relationships between individual salivary constituents and certain taste substances have been explored in human psychophysical and animal experiments. However, there are many organic and inorganic salivary constituents whose effects on taste acuity have not yet been fully elucidated. Since there is more feeling of dryness of oral cavity in mouth breathing patients the above mentioned effects do apply and hence taste perception is altered than in control group.

\section{References}

1. Harari D, Redlich M, Miri S, Hamud T, Gross M (2010) The effect of mouth breathing versus nasal breathing on dentofacial and craniofacial development in orthodontic patients. Laryngoscope 120(10): 2089-2093.

2. Barros JR, Becker HM, Pinto JA (2006) Evaluation of atopy among mouth-breathing pediatric patients referred for treatment to a tertiary care center. J Pediatr (Rio J) 82(6): 458-464.

3. McNamara JA (1981) Influence of respiratory pattern on craniofacial growth. Angle Orthod 51(4): 269-300.

4. Young T, Finn L, Kim H (1997) Nasal obstruction as a risk factor for sleep-disordered breathing. The University of Wisconsin Sleep and Respiratory Research Group. J Allergy Clin Immunol 99(2): 757-762.

5. Mojet J, Heidema J, Christ Hazelhof E (2003) Taste perception with age: generic or specific losses in suprathreshold intensities of five taste qualities? Chem Senses 28(5): 397-413.

6. Kuroishi RC, Garcia RB, Valera FC, Anselmo Lima WT, Fukuda MT (2015) Deficits in working memory, reading comprehension and arithmetic skills in children with mouth breathing syndrome: analytical cross-sectional study. Sao Paulo Med J 133(2): 78-83.

7. Bhayya DP, Shyagali TR (2009) Prevalence of oral habits in 11-13year-old school children in Gulbarga city, India. Virtual Journal of Orthodontics 8(2): 1-4.

8. Trabalon M, Schaal B (2012) It takes a mouth to eat and a nose to breathe: abnormal oral respiration affects neonates' oral competence and systemic adaptation. International Journal of Pediatrics.

9. Gudziol H, Hummel T (2007) Normative values for the assessment of gustatory function using liquid tastants. Acta Otolaryngol 127(6): 658-661.

10. Yamaguchi H, Tada S, Nakanishi Y, Kawaminami S, Shin $\mathrm{T}$, et al. (2015) Association between mouth breathing and atopic dermatitis in Japanese children 2-6 years old: a population-based cross-sectional study. PLoS One 201510: e0125916. 
11. Talavera K, Ninomiya Y, Winkel C, Voets T, Nilius B (2006) Influence of Temperature on Taste Perception. Cellular and Molecular Life Sciences.

12. Overberg J, Hummel T, Krude H, Wiegand S (2012) Differences in Taste Sensitivity Between Obese and NonObese Children and Adolescents. Arch Dis Child 97(12): 1048-1052.

13. Birch LL, Fisher JO (1998) Development of Eating behaviors Among Children and Adolescents; Pediatrics 101(3): 539-549.

14. Sifferlin A (2012) Study: Obese Kids Have Less Sensitive Taste Buds. 253: 70-78.

15. Schiffman SS, Jennifer Z (2002) Taste and Smell Perception in the Elderly: Effect of Medications and Disease. Adv Food Nutr Res 44: 247-346.

16. Brown JE, Toma RB (1986) Taste Changes during Pregnancy. Am J Clinical Nutrition 43(3): 414-418.
17. Ren E, Watari I, Jui Chin H, Kubono MM, Inoue KAP, et al. (2019) Unilateral nasal obstruction alters sweet taste preference and sweet taste receptors in rat circumvallate papillae. Acta Histochem 121(2): 135-142.

18. Wasalathanthri S, Hettiarachchi P, Prathapan S (2014) Sweet taste sensitivity in pre-diabetics, diabetics and normoglycemic controls: a comparative cross sectional study. BMC Endocr Disord 14: 67.

19. Yu J, Shin M, Kim D, Lee JR, Yoon SY, et al. (2013) Enhanced carbohydrate craving in patients with poorly controlled Type 2 diabetes mellitus. Diabet Med 30(9): 1080-1086.

20. Nanda R, Catalanotto FA (1981) Basic biological sciences long-term effects of surgical desalivation upon taste acuity, fluid intake, and taste buds in the rat. J Dent Res 60: 69-76.

21. Matsuo R (2000) Role of Saliva in the Maintenance of Taste Sensitivity. Crit Rev Oral Biol Med 11(2): 216-229. 